\title{
INVESTIGATION OF THE ECLIPSING BINARY AN TAURI
}

\author{
J.M. KREINER, J. KRZESINSKI ${ }^{1}$, G. PAJDOSZ, J. TREMKO ${ }^{2}$ and S. ZOLA ${ }^{3,1}$ \\ ${ }^{1}$ Mt.Suhora Observatory, Pedagogical University \\ ul. Podchorazych 2, 30-084 Cracow, Poland \\ ${ }^{2}$ Astronomical Institute, Slovak Academy of Sciences \\ 059-60 Tatranska Lomnica, Czechoslovakia \\ ${ }^{3}$ Astronomical Observatory, Jagiellonian University \\ ul. Orla 171, 30-244 Cracow, Poland
}

\begin{abstract}
AN Tau has been classified as the $\beta$ Lyrae-type variable. The only visual light curve was published 55 years ago. The star was observed photoelectrically during a few last years at Mt.Suhora and Skalnaté Pleso Observatories. The complete light curve was obtained in B and $\mathrm{V}$ band passes. The preliminary physical parameters of the system were obtained using the Wilson-Devinney code. From the period study of AN Tau we derived new period of the system being 1.6143843, which differs by 0.00026 from the value obtained earlier. The orbital period of AN Tau seems to be constant.
\end{abstract}

\section{Introduction}

The variable star AN Tauri $\left(B D+29^{\circ} 645\right.$, HD $281434, m=10^{m} \cdot 3-11^{m} .1$, Sp. A3) was discovered by Guthnik in 1928 (Schneller 1929). Beyer (1936) obtained long series of visual observations in 1934-1936. On the basis of his and previously published data he derived the orbital period of AN Tau equal to 1.61464. He determined also the $\beta$ Lyrae-type of the variable as well as detected a slight displacement of the secondary minimum. According to Brancewicz and Dworak (1980) both components of the system are well within their Roche lobes.

\section{Observations and Results}

The photoelectric observations of AN Tau have been carried out at the Mt.Suhora Observatory (MSO) of the Cracow Pedagogical University and at the Skalnaté Pleso Observatory (SPO) of the Slovak Academy of Sciences from February 1989 till April 1991. The twin $0.6 \mathrm{~m}$ reflecting Cassegrain telescopes were used. The observations at MSO were obtained with a double beam photometer in the $B$ and $V$ filters. The description of the photometer configuration was published by Szymanski and Udalski (1989). At SPO the telescope was equipped with a single channel photometer (its characteristics were given by Klocok et al. (1987)) and the observations were carried out with UBV filter set. $\mathrm{BD}+29^{\circ} 602$ was used as the 
comparison star at MSO while $\mathrm{BD}+29^{\circ} 647$ served as the comparison at SPO. The constancy of $\mathrm{BD}+29^{\circ} 647$ was checked occasionally against $\mathrm{BD}+29^{\circ} 633$. We found no evidence of variability of the comparison stars. The observations were corrected for differential extinction using the mean extinction coefficients for both observatories.

Unfortunately, at SPO only few observations in the $U$ and $V$ filters have been obtained, so the further analysis was based only on measurements carried out in B (MSO and SPO) and V (MSO) filters. The normal points of the observational light curve in $B$ and $V$ are shown in Fig. 1. One can see that the light curve is almost symmetrical, with a shallow secondary minimum. The ellipticity effect is clearly seen. We observed no shift of the secondary minimum, it occurred exactly in the phase 0.5 .

We derived from our observations six times of primary minima (Table 1) using graphical tracing paper method.

Table 1. Photoelectric minima of AN Tau

\begin{tabular}{cccl}
\hline HJD Min & $\sigma$ & Type & Notes \\
\hline 2447560.436 & \pm 0.002 & primary & SPO \\
7933.366 & \pm 0.001 & $"$ & MSO \\
7967.269 & \pm 0.002 & $"$ & MSO \\
8180.366 & \pm 0.002 & $"$ & SPO \\
8188.4375 & \pm 0.0010 & $"$ & MSO\&SPO \\
8206.197 & \pm 0.002 & $"$ & SPO \\
\hline
\end{tabular}

Using times of minima available in the literature as well as these listed above we computed the following new elements:

$$
\text { HJD Min } I=2428181.372( \pm 7)+1^{d} .6143843( \pm 6) \mathrm{E}
$$

This elements were used to calculate all phases in this paper.

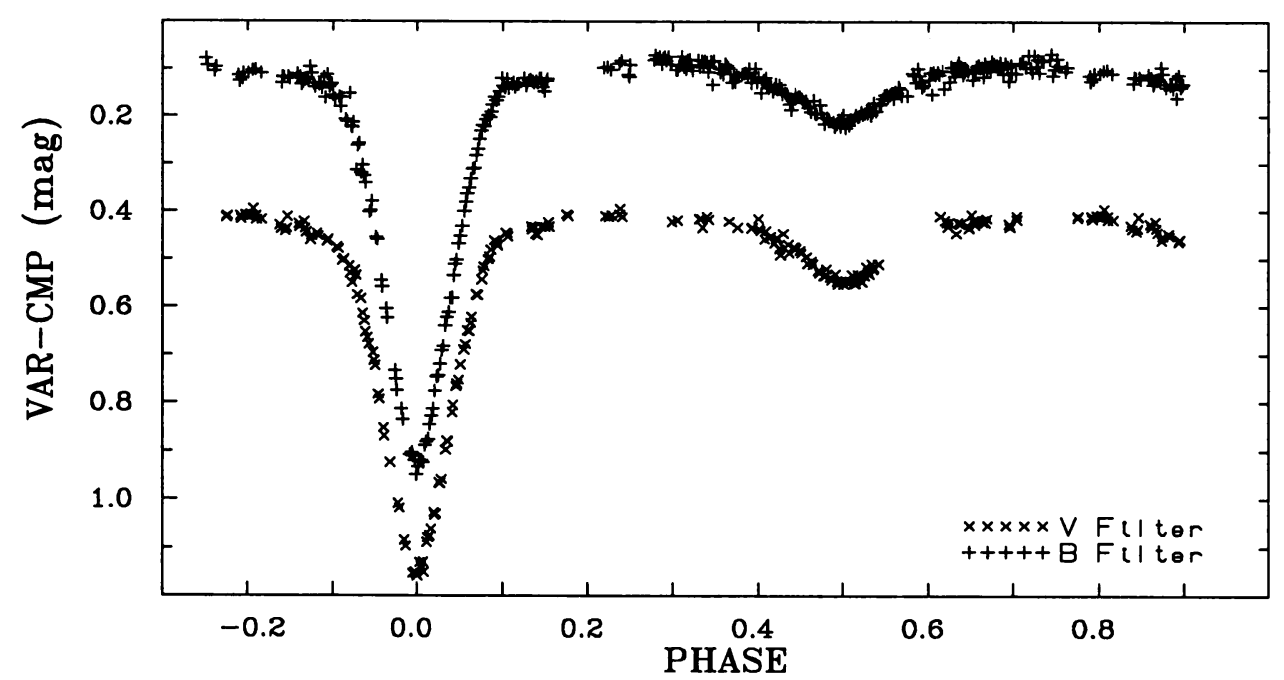

Figure 1. The light curve of $A N$ Tau in $B$ and $V$ filters. 
In our opinion there is no evidence for period changes of AN Tau, but there is a large discrepancy in some visual times of minima published by amateurs.

\section{Preliminary light curve analysis}

The Wilson-Devinney code (Wilson and Devinney 1971, Wilson 1983) was used to obtain physical parameters of AN Tau. Due to large number of unknown parameters (there is no spectroscopic observations of the system) and partial eclipses we decided to make use of the Price algorithm (Price 1976, Barone et al. 1988), which searches for the global minimum in a given range of parameters, using the Controlled Random Search (CRS) method.

We adopted the spectral type A3V of the primary star and estimated its temperature to be $8600 \mathrm{~K}$ (Popper 1980). The limb darkening coefficients were taken from Al Naimiy tables (Al Naimiy 1977). Bolometric albedos and gravity darkening coefficients of components were set to their theoretical values. The following ranges of parameters were searched in the CRS method: $60^{\circ} \leq i \leq 90^{\circ}, \quad 0.1 \leq q \leq 5.0,4000 \leq T 2 \leq 7000 \mathrm{~K}$, $1 \leq \Omega_{1,2} \leq 12, \quad 3 \leq L_{1} \leq 12, \quad 0 \leq \ell 3 \leq 0.35$.

Iterations were performed until the difference between the best and the worst values of the sum of squares of residuals of the search array elements was smaller than $15 \%$. Results are shown in Fig. $2 a(\Omega 2-q$ plane), Fig. 2b ( $\Omega_{1}-q$ plane) and Fig. 2c ( $i-q$ plane). We found that for $q<0.9$ the secondary component in the system is very close to or even fills its Roche lobe (see Fig. 2a). For $q>0.9$ the system is evidently detached.
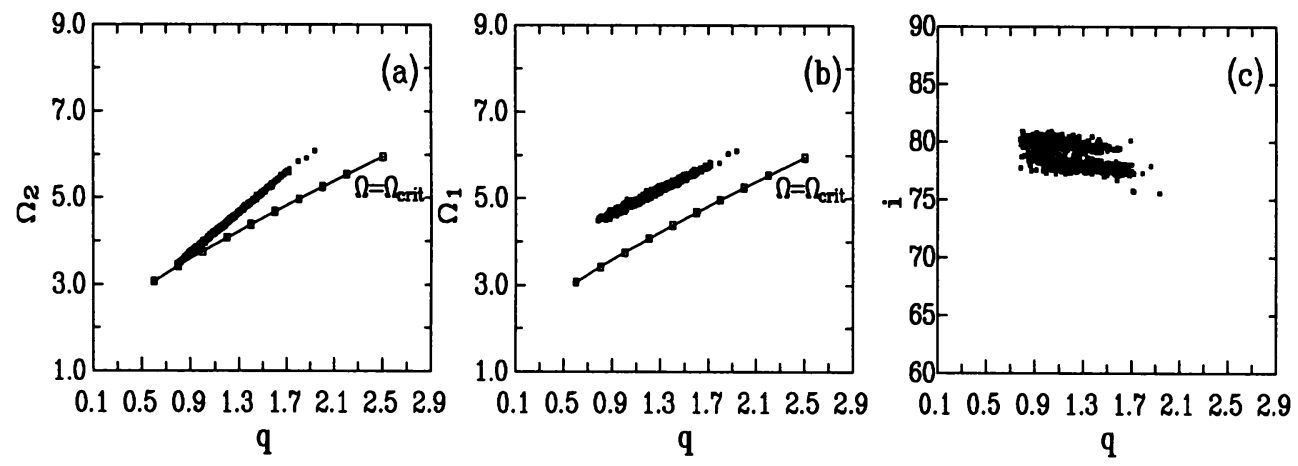

Figure $2 a, b$ and $c$. Graphical representation of the search array.

Our final step was to find the best solution for semidetached $(q<0.9)$ and detached $(q>0.9)$ configuration. We used the differential correction method provided by the Wilson-Devinney code. The results for both configurations are presented in Table 2.

\section{Conclusions}

We have made the first attempt to determine physical parameters of AN 
Tau. Due to large number of free parameters, strong correlations between them and not reliably determined temperature of the primary star, the obtained results should be considered as preliminary. The following reasons could explain the existence of a large amount of the resulting third light:

- background stars (Hilditch and Hill (1975) reported a faint star near the system) or a real third component,

- an incorrect value of the primary component temperature used in calculations.

Spectroscopic observations, particularly velocity light curve could verify our solution and give more information about the nature of the system.

Table 2. Photometric solutions.

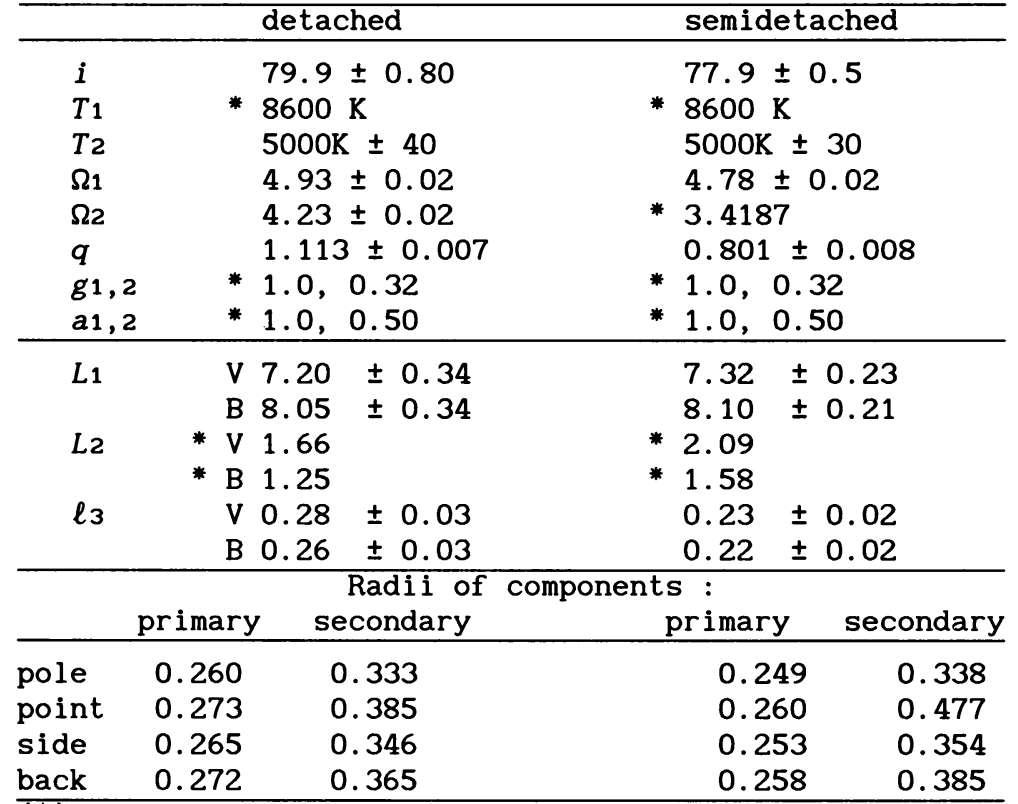

(*) - not adjusted

REFERENCES:

Al Naimiy H.M.: 1977, Astrophys.Space Sci. 53, 181.

Barone F., Maceroni C., Milano L., Russo G.: 1988, Astr. Astrophys. 197, 347.

Beyer M.: 1936, Astr.Nachr. 260, 11.

Brancewicz H.K., Dworak T.Z.: 1980, Acta Astr. 30, 501.

Hilditch R.W., Hill G.: 1975, Mem.R.Astr.Soc. 79, 101.

Klocok L., Žižnovsky J., Zverko J.:1987, Contr.Obs.Skalnate Pleso 16, 43.

Popper D.M.: 1980, Ann. Rev. Astr.Astrophys. 18, 115.

Price W.L.: 1976, Computer J. 20, 367.

Schneller H.: 1929, Astr. Nachr. 235, 85.

Szymanski M., Udalski A.: 1989, Acta Astr. 39, 1.

Wilson R.E.: 1983, Astrophys.Space Sci. 92, 229.

Wilson R.E., Devinney E.J.: 1971, Astrophys.J. 166, 605. 